BIOMEDICA

Vol. 1, No. $4-1981$

\title{
AISLAMIENTO DE TRES CEPAS DE LEISHMANIA A PARTIR DE LUTZOMYIA TRAPIDOI EN COLOMBIA
}

\author{
ALBERTO MORALES. ${ }^{*}$ AUGUSTO CORREDOR. ${ }^{\star *}$ ELVIA CACERES. ${ }^{* * *}$ ANA LUCIA IBAGOS. ${ }^{* \star * \star}$ \\ CRISTINA DE RODRIGUEZ.*****
}

\begin{abstract}
En un foco de Leishmaniasis Cutánea en un bosque en la vereda Flor Azul, municipio de Mariquita, Departamento del Tolima, Colombia, se recolectaron, usando trampas de luz (C D C), trampas Shannon, cebo humano, cebo animal (caballo) y captura en huecos de árboles, de febrero de 1979 a mayo de 1981, un total de 4.081. $\delta^{\prime} \delta^{\prime}$ y $7.912 \uparrow q$ de Lutzom yia (Diptera, Psychodidae), las cuales pertenecían a 19 especies. De las 7.912 hembras recolectadas se hizo disección del tubo digestivo a 3.337 multíparas en busca de flagelados, los cuales se encontraron en: una Lutzomyia shannoni, una Lutzom yia gomeziy cuarenta y dos Lutzomyia trapidoi. De estos 44 intestinos con flagelados se inocularon 32 hamsters, con el siguiente resultado: 2 hamsters murieron por causas desconocidas lel de L. shannoni y $L$. gomezh; de $\mathbf{3 0}$ hamsters inoculados con flagelados hallados en intestino de L. trapidoi, tres fueron positivos para Leishmanai, 12 permanecen en observación y son negativos hasta la fecha y $\mathbf{1 5}$ murieron por causas desconocidas. Las tres cepas de Leishmania aisladas de L. trapidoise mantienen en hamsters y en cultivos en medio de las $4 \mathrm{~N}$.
\end{abstract}

Del total de 2.869 hembras de Lutzomyia trapidoi disectadas, 2.356 eran multíparas; de éstas se encontraron $\mathbf{4 2}$ con flagelados en el intestino. Por la localización de los flagelados en el intestino anterior de L. trapidoi, en una de las cepas aisladas, por el tan corto período de incubación de las cepas inoculadas en el hamster y por la facilidad con la cual se cultivan, es posible pensar que estas cepas pertenezcan al complejo Leishmania mexicana.

Esta es la primera vez que en Colombia se aislan cepas de Leishmania del intestino del insecto vector.

\footnotetext{
* Bacteriólogo, MSc. Grupo de Entomología, Instituto Nacional de Salud (INS). Apartado 80334. Bogotá, Colombia.

** Médico. Grupo de Parasitología (INS).

*** Bacterióloga. Grupo de Parasitología (INS).

**** Bióloga. Grupo de Entomología (INS).

***** Bióloga. Grupo de Entomología (INS).
} 


\section{INTRODUCCION}

El 20 de julio de 1978 cuatro hombres fueron de cacería a un bosque de la vereda Flor Azul, Municipio de Mariquita, Departamento del Tolima, donde permanecieron durante toda la noche y según relataron posteriormente, fueron picados por una enorme cantidad de "capotillos" (término vernáculo, entre otros, con que se designa a los insectos del género Lutzomyia en Colombia). Tres de estas personas tenían como residencia habitual la vereda Quebradahonda, contigua a la vereda de Flor Azul y la cuarta había vivido durante varios años en el área urbana de la población de Mariquita.

Poco tiempo después estos cuatro hombres desarrollaron úlceras cutáneas y el 22 de septiembre de 1978, el Grupo de Parasitología, del Instituto Nacional de Salud les practicó frotis directo de las lesiones en busca de Leishmania, con resultado positivo. Este hallazgo indicaba que en aquel bosque había una alta transmisión de Leishmania. Una encuesta epidemiológica posterior, realizada a los residentes en casas vecinas al bosque mencionado, reveló que había alguna incidencia de úlceras activas y cicatrices, debidas probablemente a Leishmania. Se decidió entonces realizar en esa área un estudio epidemiológico a largo plazo que comprendiera la población humana, el artrópodo vector, el parásito y el reservorio. Los estudios respectivos se iniciaron en febrero de 1979 y este trabajo es un informe del aislamiento de tres cepas de Leishmania, que parasitaban a Lutzomyia trapidoi (Fairchild \& Hertig, 1952) (Diptera, Psychodidae), en el foco de Leishmaniasis Cutánea en la vereda Flor Azul, Mariquita, Tolima.

\section{MATERIALES Y METODOS}

\section{Area de Estudio:}

La Vereda Flor Azul está situada al oeste de la población de Mariquita, en un área montañosa con pequeños y estrechos valles surcados por algunas corrientes de agua. El terreno está dedicado a la agricultura y la ganadería; se cultiva especialmente café, caña y cacao. Aunque es posible encontrar café de la variedad llamada "caturra", que no necesita sombrío, la mayor parte de los cafetales tienen entremezclados árboles de varias especies y tamaños, algunos de gran altura, además de matas de plátano.

De vez en cuando se hallan pequeñas manchas de bosques, que han sido conservadas como reservas de leña para las hornillas en la producción de la panela, para su utilización en el cercado de potreros, etc. En uno de estos bosques fue donde se infectaron con Leishmania los cuatro cazadores mencionados, se hicieron la mayor parte de las capturas de Lutzomyia y se aislaron las tres cepas de Leishmania. Este bosque (Fig. 1), con un área aproximada de ocho hectáreas, está situado exactamente en el vértice del triángulo que forma la desembocadura de la quebrada Cachipay en el Río Sucio; constituido por bosque secundario, aunque conserva algunos árboles que son restos de bosque primitivo, está rodeado principalmente por potreros con rastrojo y en mínima parte por cafetales. El declive de su terreno es muy pronunciado. Dentro de él no existe ninguna habitación humana.

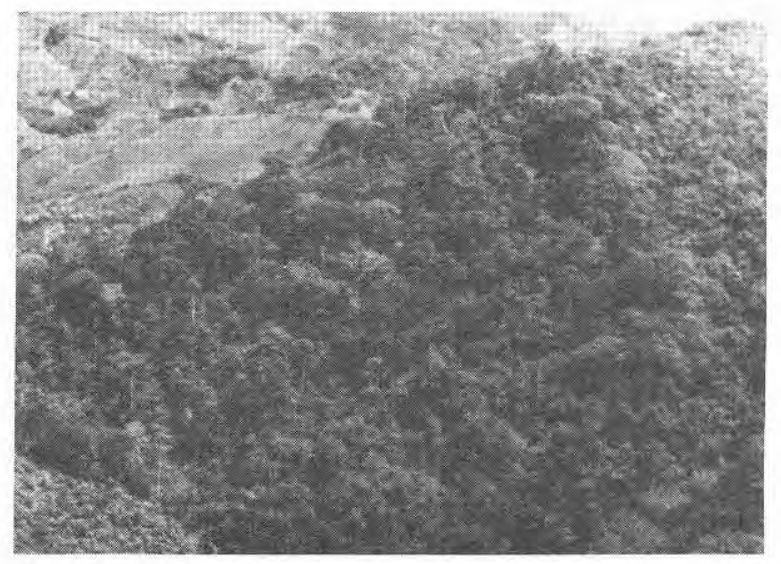

Fig. 1. Bosque húmedo tropical de la vereda Flor Azul, Mariquita, Tolima, en donde se capturaron las tres hembras de L. trapidoi, infectadas con promastigotes.

La vereda Flor Azul está localizada, de acuerdo a la clasificación de Holdrige, en área de bosque húmedo tropical. (1).

\section{Encuesta Entomológica:}

En febrero de 1979 se inició la búsqueda de Lutzomyia con el propósito de obtener 
una información general de las especies que se encuentran en el área. Los insectos se buscaron en los sitios de reposo en huecos de árboles y utilizando cebo humano, cebo animal (caballo), trampas de luz tipo C.D.C. y trampa Shannon; los flebotomíneos capturados se mataron con cloroformo y se conservaron en seco, hasta establecer su identificación en el laboratorio en Bogotá. Para ésta, los ejemplares machos y hembras, por separado, eran introducidos por unos segundos en un detergente al $2 \%$ en solución salina y pasados inmediatamente a solución de Nesbitt (2), en donde permanecían de un día para otro o más tiempo, si era necesario. Una vez aclarados eran disectados y montados entre lámina y laminilla con medio de Berlese (3), procediendo luego a su determinación específica.

\section{Búsqueda de Promastigotes en los Flebotomíneos:}

Los insectos eran atrapados con aspiradores que funcionaban con baterías de linterna y de allí eran transferidos a tarugos de guadua o bambú; estos eran trasladados a un termo metálico que en su fondo tenía una capa de hielo de agua. Inicialmente, los tarugos de guadua conteniendo los insectos, eran llevados al laboratorio y colocados en nevera; al día siguiente las hembras eran disectadas en busca de flagelados. Posteriormente se cambió este método por el de la conservación de los insectos en nitrógeno líquido, de acuerdo con la técnica de Minter y Goedbloed (4), en seguimiento de la cual, las hembras eran sacadas de los tarugos de guadua con un aspirador, pasadas por unos segundos en un detergente al $2 \%$ en solución salina, para hidratarlas e inmediatamente introducidas en viales de policarbonato que contenían un medio protector o de soporte, compuesto de solución salina fosfatada amortiguada (P B S) de $\mathrm{pH} 7.4$ y dimetil sulfóxido (D M S O), en la proporción de $90 \%$ de P.B.S. y $10 \%$ de D M S O . Nunca eran introducidas más de 30 hembras de Lutzomyia en cada vial. Los viales conteniendo las hembras eran colocados dentro de un tubo de vidrio que se cubría con plastilina. Estos tubos, así preparados, eran expuestos a los vapores de nitrógeno líquido durante 15-20 minutos e inmediatamente colocados en los soportes metálicos del termo de nitrógeno, en donde permanecían hasta el día en que se realizaba la disección del tubo digestivo de las hembras en busca de los flagelados. Para la disección de las hembras, se sacaban los viales del nitrógeno líquido y se colocaban en un baño de agua a $40^{\circ} \mathrm{C}$. para descongelación del medio protector. Una vez descongelado el medio, se colocaban las hembras individualmente en solución salina isotónica sobre un porta-objetos y se extraía el tubo digestivo, con la ayuda visual de un microscopio estereoscópico. El tubo digestivo era examinado luego, bajo microscopio de luz en contraste de fase. Cuando se encontraban flagelados, se maceraba el tubo digestivo con ayuda de agujas, se recogía este producto en $0,2 \mathrm{ml}$. de solución salina en una jeringa de tuberculina y se inoculaba intradérmicamente en la naríz de un hamster.

Cuando era posible, se anotaba la localización de los protozoarios en el tubo digestivo de las hembras positivas para flagelados; además se buscaban los protozoarios en la faringe y la proboscis. A algunas hembras se les examinaban las glándulas accesorias para determinar si eran nulíparas o multíparas. En el momento de la disección se hacía la determinación específica de las especies de Lutzomyia.

\section{Aislamiento de las Cepas de Leishmania:}

Los hamsters inoculados con los flagelados encontrados en el tubo digestivo de los insectos fueron sometidos a observación y cuando aparecieron signos de eritema y edema en la naríz se procedió a hacer frotis directo con la linfa extraída por punción, para investigar formas amastigotas. En los casos positivos, en que la preparación mostraba varias formas por campo, la linfa se inoculó en nuevos hamster y se sembró en el medio de cultivo de las 4 N. Se determinó que si la preparación mostraba menos de una forma amastigota por campo, se continuaría la observación hasta cuando se presentaran varias formas por campo.

\section{RESULTADOS}

De febrero de 1979, mes de iniciación del programa, a mayo 30 de 1981 , se recolectó 
un total de 11.993 especímenes de Lutzomyia,

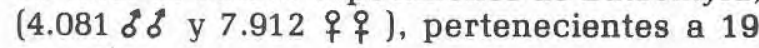
especies.

En el cuadro No. 1 se muestran los resultados de la recolección de machos. Solo de tres de las 19 especies de Lutzomyia encontradas en el área no se capturaron machos: Lutzomyia cercanas a atroclavata, ovallesiy serrana. Como era de esperarse, la mayoría de los machos fueron capturados en los sitios de reposo en los huecos de los árboles. El hecho de capturarse machos con cebo humano se debe a que se acercan tratando de copular con las hembras de Lutzomyiaque son atraídas por el cebo o por la luz de la linterna que usa el capturador.

\section{CUADRO № ।}

MACHOS DE LUTZOMYIA RECOLECTADOS EN EL FOCO DE LEISHMANIASIS CUTANEA DE MARIQUITA-TOLIMA

\begin{tabular}{|c|c|c|c|c|c|c|}
\hline ESPECIE & $\begin{array}{c}\text { CEBO } \\
\text { HUMANO }\end{array}$ & $\begin{array}{l}\text { TRAMPA } \\
\text { DE LUZ } \\
\text { (CDC) }\end{array}$ & $\begin{array}{l}\text { TRAMPA } \\
\text { SHANNON }\end{array}$ & $\begin{array}{c}\text { CEBO } \\
\text { ANIMAL } \\
\text { CABALLO }\end{array}$ & $\begin{array}{c}\text { HUECO } \\
\text { DE } \\
\text { ARBOL }\end{array}$ & TOTAL \\
\hline ㄴ. barrettoi & - & 5 & - & - & - & 5 \\
\hline L. bifoliato & 3 & - & - & - & 223 & 226 \\
\hline L. gomezi & 11 & 5 & 5 & - & 37 & 58 \\
\hline L. hartmanni & 30 & 15 & 24 & 6 & 9 & 84 \\
\hline L. lichyi & 5 & - & 2 & - & 2.525 & 2.532 \\
\hline L. nordestina & 1 & - & - & - & 2 & 3 \\
\hline L. panamensis & 2 & - & - & - & - & 2 \\
\hline ㄴ. pilosa & - & - & - & - & 11 & 11 \\
\hline L. soulensis & - & - & 1 & - & - & 1 \\
\hline L. shannoni & 66 & 8 & 28 & - & 511 & 613 \\
\hline L. spinosa & 1 & - & - & - & 4 & 5 \\
\hline L. trapidoi & 230 & 24 & 17 & 1 & 47 & 319 \\
\hline$\underline{\text { L. }}$ trinidadensis & - & - & 8 & 1 & 94 & 103 \\
\hline L. triramula & - & 5 & - & - & 2 & 7 \\
\hline 느. vespertilionis & 18 & 2 & 3 & - & 38 & 61 \\
\hline ㄴ. walkeri & 2 & I & 1 & 1 & 46 & 51 \\
\hline TOTAL & 369 & 65 & 89 & 9 & 3.549 & 4.081 \\
\hline
\end{tabular}

Con los diferentes métodos de captura empleados se recolectó un total de 7.912 hembras pertenecientes a 18 especies (cuadro No. 2).

De las 19 especies encontradas en el área no se capturó ninguna hembra de Lutzomyia triramula.
CUADRO № 2

HEMBRAS DE LUTZOMYIA RECOLECTADAS EN EL FOCO DE LEISHMANIASIS CUTANEA DE MARIQUITA - (TOLIMA)

\begin{tabular}{|c|c|c|c|c|c|c|}
\hline ESPECIE & $\begin{array}{c}\text { CEBO } \\
\text { HUMANO }\end{array}$ & $\begin{array}{c}\text { TRAMPA } \\
\text { DE LUZ } \\
(C D C)\end{array}$ & $\begin{array}{l}\text { TRAMPA } \\
\text { SHANNON }\end{array}$ & $\begin{array}{c}\text { CEBO } \\
\text { ANIMAL } \\
\text { CABALLO }\end{array}$ & $\begin{array}{l}\text { HUECO } \\
\text { DE } \\
\text { ARBOL }\end{array}$ & TOTAL \\
\hline $\begin{array}{l}\text { L. cercana a } \\
\text { atroclavata }\end{array}$ & - & - & 1 & - & - & 1 \\
\hline L. barrettoi & - & 3 & 6 & - & - & 9 \\
\hline ㄴ. bifoliata & 37 & - & - & - & 11 & 48 \\
\hline ㄴ. gomezi & 487 & 61 & 36 & - & 55 & 639 \\
\hline ㄴ. hartmanni & 525 & 48 & 57 & 25 & 30 & 685 \\
\hline ㄴ. lichyi & 180 & 20 & 1 & 25 & 702 & 928 \\
\hline 느. nordestina & - & - & - & - & 4 & 4 \\
\hline L. ovallesi & - & - & 1 & - & 1 & 2 \\
\hline 느, panamensis & 8 & 4 & 1 & - & - & 13 \\
\hline ㄴ. pilosa & 8 & - & - & - & 4 & 12 \\
\hline 느 saulensis & - & - & 4 & - & - & 4 \\
\hline L. serrana & - & - & - & - & 3 & 3 \\
\hline L. shannoni & 75 & 11 & 6 & - & 159 & 251 \\
\hline ㄴ. spinosa & 1 & - & - & - & 2 & 3 \\
\hline ㄴ. trapidoi & 3.681 & 324 & 282 & 55 & 428 & 4.770 \\
\hline ㄴ. trinidadensis & 1 & - & 4 & - & 4 & 9 \\
\hline ㄴ. vespertilionis & - & 2 & - & - & - & 2 \\
\hline ㄴ.. walkeri & 1 & 3 & 8 & - & 19 & 31 \\
\hline Lutzomyia ssp & 498 & - & - & - & - & 498 \\
\hline TOTAL & 5.502 & 476 & 407 & 105 & 1.422 & 7.912 \\
\hline
\end{tabular}

Nuestros resultados obtenidos concuerdan con el hecho, ya bien conocido, que las hembras de L. gomezi, L. hartmanni, L. lichyi y L. trapidoi son altamente antropofílicas. Se recolectó una apreciable cantidad de hembras de Lutzomyia shannoni con cebo humano (75 de 251); ésta es una especie que se alimenta en aves, reptíles, anfibios y mamíferos, incluído el hombre.

Es interesante anotar que fueron recolectadas con cebo humano 37 hembras de L. bifoliata, siendo esta la primera vez, hasta donde nosotros sabemos, que se tiene información de la antropofilia de esta especie. En la columna correspondiente a las hembras recolectadas con cebo humano aparecen 489 Lutzomyia spp., que no pudieron separarse por especie debido a que en el momento de su captura no se disponía de nitrógeno líquido y fue necesario homogeneizarlas en conjunto por la carencia de tiempo para disectarlas individualmente; sin embargo, al examinar el producto de esta homogeneización, no se observó ningún flagelado. 
En cuanto a L. trapidoi, se recolectó un total de 4.770 hembras, de las cuales 3.681 lo fueron con cebo humano, 324 con trampa de luz (C D C), 282 con trampa Shannon, 55 con cebo animal (caballo) y 428 en huecos de árboles en los sitios de reposo.

En el cuadro No. 3 se muestra, por especies, el número de hembras a las cuales se les practicó disección del tubo digestivo en busca de flagelados. Se disectaron 3.928 hembras de las cuales 44 fueron positivas, para éstos, así: una de 383 Lutzomyia gomezi, una de 78 Lutzomyia shannoni y 42 de 2.869 Lutzomyia trapidoi. Esto significa que para L. trapidoi la proporción de hembras con flagelados fue de 1 por cada 68,3 disectadas.

Los flagelados encontrados en $L$. shannoni y L. gomezi fueron inoculados en hamsters, pero éstos murieron a las 8 y 22 semanas, por causas desconocidas. Del total de 2.869 hembras de Lutzomyia trapidoi disectadas 2.536 eran multíparas, en las cuales se encontraron 42 con flagelados en el intestino. De estas 42, se inocularon en hamster los macerados intestinales de 30; no se inocularon los de las 12 restantes debido a que el número de flagelados en su intestino era muy escaso. De 3 de los 30 hamsters inoculados se aislaron cepas de parásitos similares a Leishmania; de los 27 hamsters restantes, 15 murieron por causas desconocidas y 12 se mantienen en observación.

La historia del aislamiento de estas cepas puede verse en el cuadro No. 4. Todas ellas provenían de hembras de L. trapidoi recolectadas en el bosque en donde se infectaron los cuatro cazadores, en capturas nocturnas con cebo humano y conservadas en nitrógeno líquido. La hembra capturada el 6 de noviembre de 1980 fue disectada el 23 de diciembre de 1980 y sus flagelados inoculados inmediatamente al hamster. La hembra recolectada el 4 de febrero de 1981 fue disectada el 5 de marzo de 1981 y la inoculación se hizo el mismo día. La tercera hembra, capturada en febrero 9 de 1981 fue disectada y practicada la inoculación en marzo 17 de 1981.

El 6 de mayo de 1981 se comprobó, por frotis directo de la linfa, que los tres hamsters eran positivos para formas amas- tigotas (Fig. 2) y entonces se hicieron pases a nuevos hamsters y siembras en medio de las $4 \mathrm{~N}$.

CUADRO № 3

HEMBRAS DE LUTZOMYIA DISECTADAS EN BUSCA DE FLAGELADOS-VEREDA FLOR AZUL-MARIQUITA (TOLIMA)

\begin{tabular}{|c|c|c|c|c|c|c|}
\hline \multirow{2}{*}{ ESPECIE } & \multirow{2}{*}{ CANTIDAD } & \multirow{2}{*}{$\begin{array}{l}\text { MULTI- } \\
\text { PARAS }\end{array}$} & \multirow{2}{*}{$\begin{array}{l}\text { NULI - } \\
\text { PARAS }\end{array}$} & \multirow{2}{*}{$\begin{array}{l}\text { SIN ESPE } \\
\text { CIFICAR }\end{array}$} & \multicolumn{2}{|c|}{ FLAGELADOS } \\
\hline & & & & & Negativos & Positivas \\
\hline ㄴ. gomezi & 383 & 268 & 5 & 110 & 382 & 1 \\
\hline L. hartmanni & 409 & 317 & 4 & 88 & 409 & - \\
\hline L. lichyi & 177 & 136 & 1 & 40 & 177 & - \\
\hline ㄴ. nordestina & 1 & 1 & - & - & 1 & - \\
\hline ㄴ. panamensis & 5 & 5 & - & - & 5 & - \\
\hline ㄴ. shonnoni & 78 & 68 & - & 10 & 77 & 1 \\
\hline ㄴ. trapidoi & 2.869 & 2.536 & 47 & 286 & 2.827 & 42 \\
\hline ㄴ. trinidadensis & 1 & 1 & - & - & 1 & - \\
\hline ㄴ. vespertilionis & 1 & 1 & - & - & 1 & - - \\
\hline ㄴ. walkeri & 4 & 4 & - & - & 4 & - \\
\hline TOTAL & 3.928 & 3.337 & 57 & 534 & 3.884 & 44 \\
\hline
\end{tabular}

CUADRO № 4

CEPAS DE LEISHMANIA AISLADAS DE LUTZOMYIA TRAPIDOI MARIQUITA (TOLIMA)

\begin{tabular}{|c|c|c|c|c|c|}
\hline $\begin{array}{l}\text { FECHA DE } \\
\text { CAPTURA } \\
\text { INSECTO }\end{array}$ & CEBO & CONSERVACION & $\begin{array}{l}\text { INOCULACION } \\
\text { AL HAMSTER }\end{array}$ & \multicolumn{2}{|c|}{$\begin{array}{l}\text { POSITIVIDAD } \\
\text { EN HAMSTER }\end{array}$} \\
\hline Noviemb. $6 / 80$ & Humano & $\begin{array}{l}\text { Nitrógeno } \\
\text { Liquido }\end{array}$ & Diciemb. $23 / 80$ & Mayo & $6 / 81$ \\
\hline Febrero $4 / 81$ & Humano & $\begin{array}{l}\text { Nitrógeno } \\
\text { Líquido }\end{array}$ & Marzo $5 / 81$ & Mayo & $6 / 81$ \\
\hline Febrero $9 / 81$ & Humano & $\begin{array}{l}\text { Nitrógeno } \\
\text { Liquido }\end{array}$ & Marzo $17 / 81$ & Mayo & $6 / 81$ \\
\hline
\end{tabular}

En el cuadro No. 5 se muestra cómo evolucionaron en el hamster las tres cepas aisladas. Al hamster inoculado con la cepa No. 1 se le observó eritema y edema nasales el día 56 post-inoculación con los promastigotes (Fig. No. 2); al de la cepa No. 2, el día 46 y al de la cepa No. 3 el día 34 . Seguramente, si en este momento se hubiera hecho un frotis directo, se habrían podido demostrar las formas amastigotas, sin embargo, éste solo se realizó el 6 de mayo, a los 134, 62 y 50 días de haberse hecho la 
inoculación con las cepas 1,2 y 3, respectivamente. De las tres cepas se han hecho ya dos pases en hamster, con los resultados que se pueden ver en el citado cuadro No. 5 . Por otra parte las tres cepas se mantienen cultivadas en medio de las $4 \mathrm{~N}$ (Fig. 4).

En el caso de la cepa No. 1 se había logrado establecer la localización de los flagelados en los intestinos medio y anterior del tubo digestivo de la hembra de L. trapidoi para las otras 2 cepas no se pudo establecer la localización en el intestino. En ninguna de las tres cepas se observaron flagelados, $\mathrm{ni}$ en la faringe ni en la proboscis del insecto vector.

Por la localización de los flagelados en el tubo digestivo (5), observada en una de las hembras de L. trapidoi positivas para Leishmania; por la facilidad con que cultivaron las tres cepas y por el relativamente corto período de incubación en el hamster, es posible pensar que estas cepas pertenezcan al complejo Leishmania mexicana; será necesario, para tipificarlas, recurrir a métodos bioquímicos.
CUADRO № 5

EVOLUCION EN EL HAMSTER DE 3 CEPAS DE LEISHMANIA AISLADA DE LUTZOMYIA TRAPIDOI - MARIQUITA (TOLIMA)

\begin{tabular}{|c|c|c|c|c|c|c|c|}
\hline \multirow{3}{*}{$\begin{array}{l}\text { CEPA } \\
\text { № }\end{array}$} & \multirow{3}{*}{$\begin{array}{c}\text { FECHA DE } \\
\text { INOCULACION } \\
\text { PROMASTIGOTES }\end{array}$} & \multirow{3}{*}{\begin{tabular}{|l} 
DIAS \\
ERITEMA \\
EDEMA
\end{tabular}} & \multirow{3}{*}{$\begin{array}{l}\text { POST - I } \\
\text { AMASTI- } \\
\text { GOTAS } \\
\text { FROTIS } \\
\text { DIRECTO } \\
\end{array}$} & \multirow{2}{*}{\multicolumn{2}{|c|}{$\begin{array}{c}\text { NOCULACION PR } \\
\text { Primer Pase }\end{array}$}} & OMASTI & SOTES \\
\hline & & & & & & \multicolumn{2}{|c|}{ Segundo Pase } \\
\hline & & & & $\begin{array}{c}\text { Eritema } \\
\text { Edema }\end{array}$ & $\begin{array}{l}\text { Amasti- } \\
\text { gotas }\end{array}$ & $\begin{array}{l}\text { Eritema } \\
\text { Edema }\end{array}$ & $\begin{array}{l}\text { Amasti- } \\
\text { gotus }\end{array}$ \\
\hline 1 & Diciemb. $23 / 80$ & 56 & 134 & 38 & 68 & 29 & 35 \\
\hline 2 & Marzo & 46 & 62 & 41 & 75 & - & - \\
\hline 3 & Morzo $17 / 81$ & 34 & 50 & 28 & 84 & 22 & - \\
\hline
\end{tabular}

En Panamá, L. trapidoi ha sido incriminada como vector de Leishmania braziliensis panamensis (6-7). En Colombia, hasta donde nosotros sabemos, es la primera vez que se aislan cepas de Leishmania a partir de un insecto vector. En la vereda Flor Azul, entre las 19 especies encontradas hasta ahora en el área, L. trapidoi es una de las más abundantes, altamente antropofílica, pica además a caballares y se recolecta en abundancia en el peridomicilio humano.

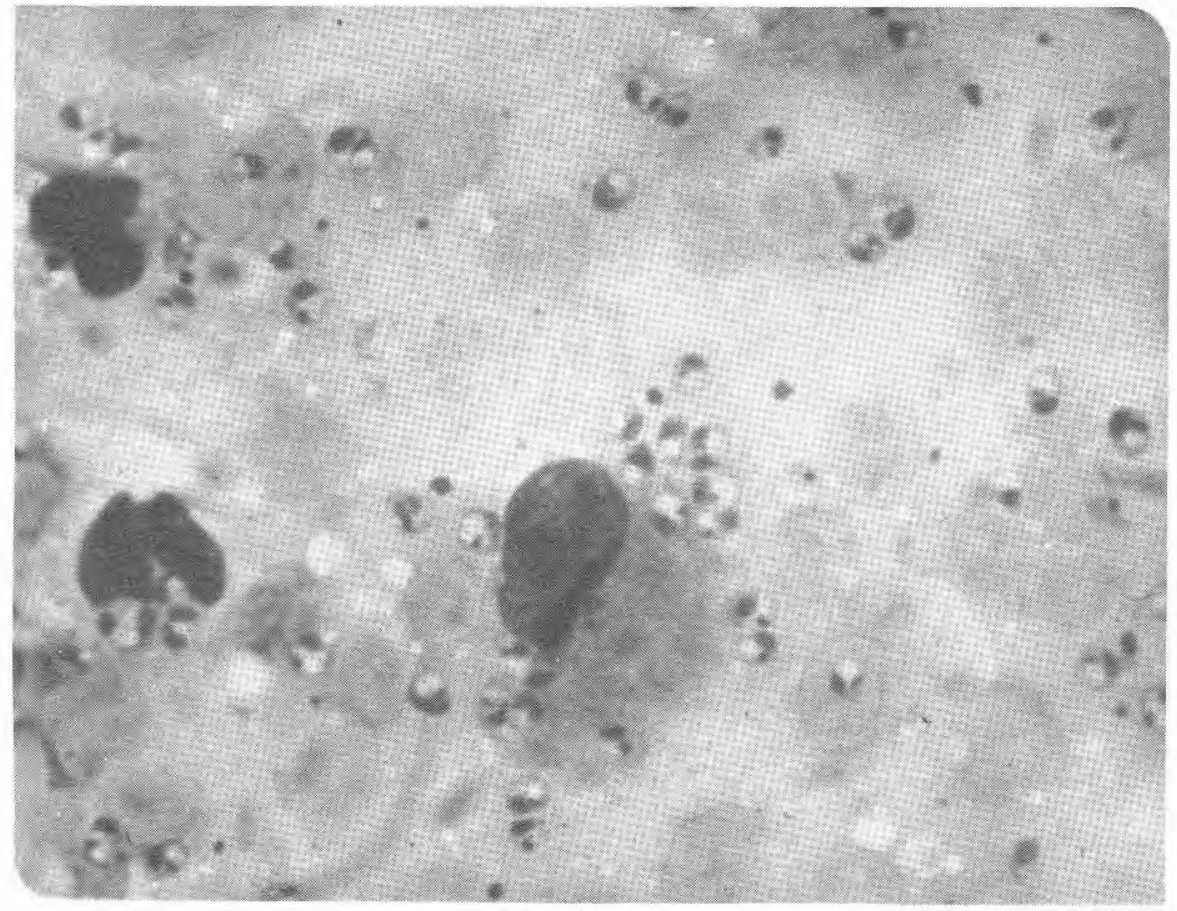

Fig. 2. Amastigotes de Leishmania $s p$. provenientes de extendido directo de la nariz de un hamster inoculado con promastigotes encontra. dos en un flebotomineo naturalmente infectado, L. trapidoi. 


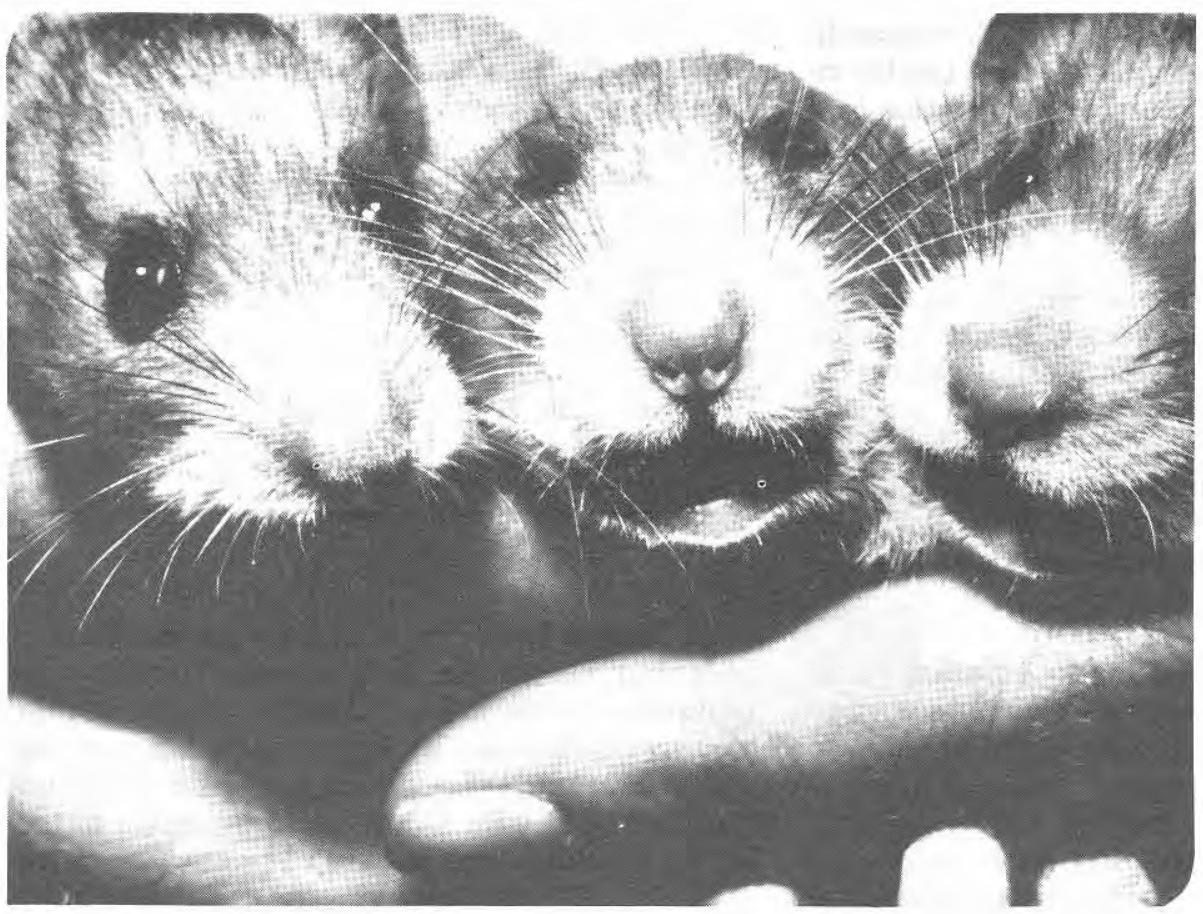

Fig. 3. Hamsters inoculados intra. dérmicamente en la nariz, con pro mastigotes prove. nientes del intes. tino medio de L. trapidoi. Obsér vese el acentuado edema con turgen cia de la nariz.

Fig. 4. Promastigo. tes en cultivo a par. tir de la biopsia nasal de un hamster inoculado con pro. mastigotes hallados en L. trapidoi.

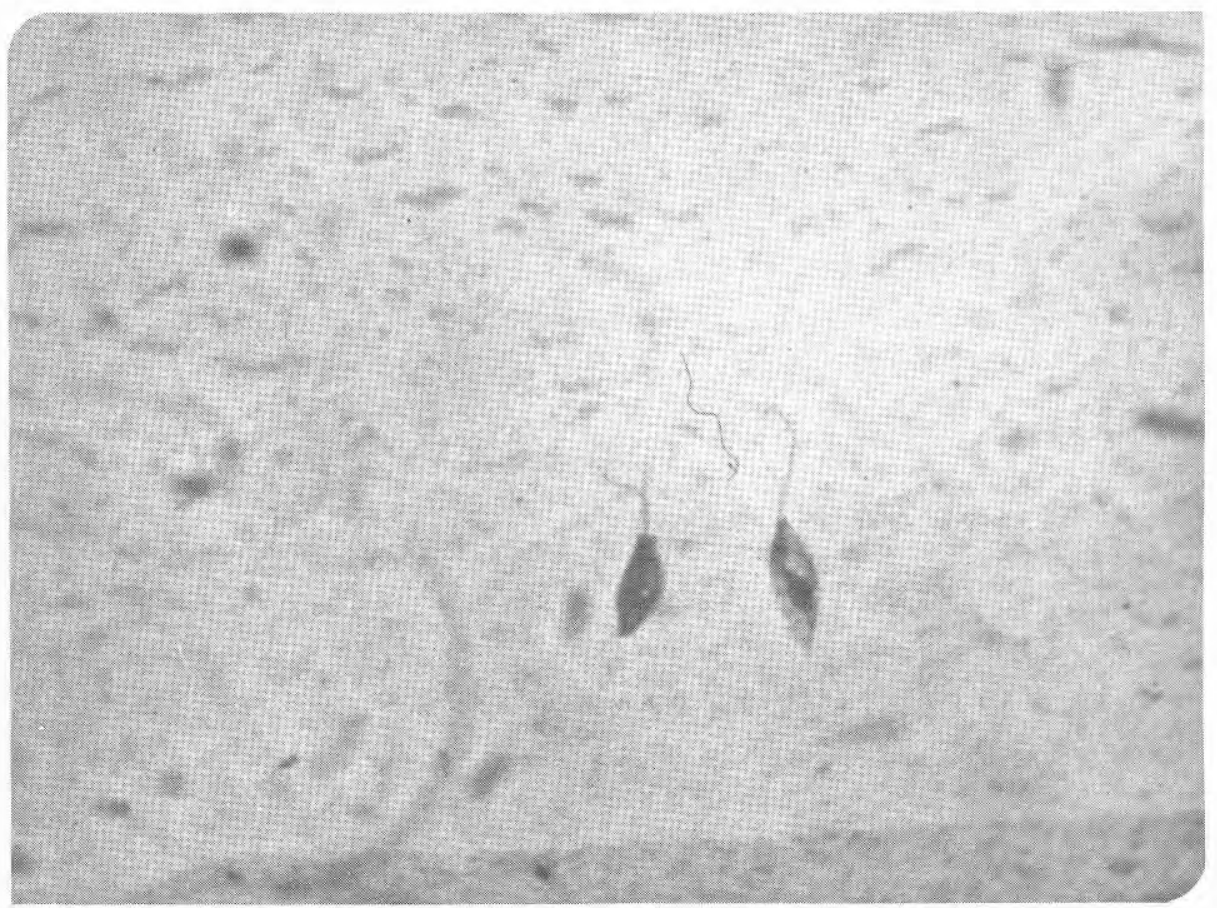


En la figura No. 5 se muestra la distribución conocida de L. trapidoi en Colombia (8-9-10). Esta especie ha sido hallada en Antioquia: Río Anorí, Mutatá (Río Porroso) y Chigorodó (Currulao); Boyacá: Puerto Boyacá; Chocó: Alto Curiche, Teresita y Río Atrato (Sautatá); Santander: San Vicente de Chucurí; Tolima: Mariquita (Fátima y Flor Azul); Valle: Anchicayá, Buenaventura, Río Raposo, Hogar del Niño y Palmeras del Pacífico.

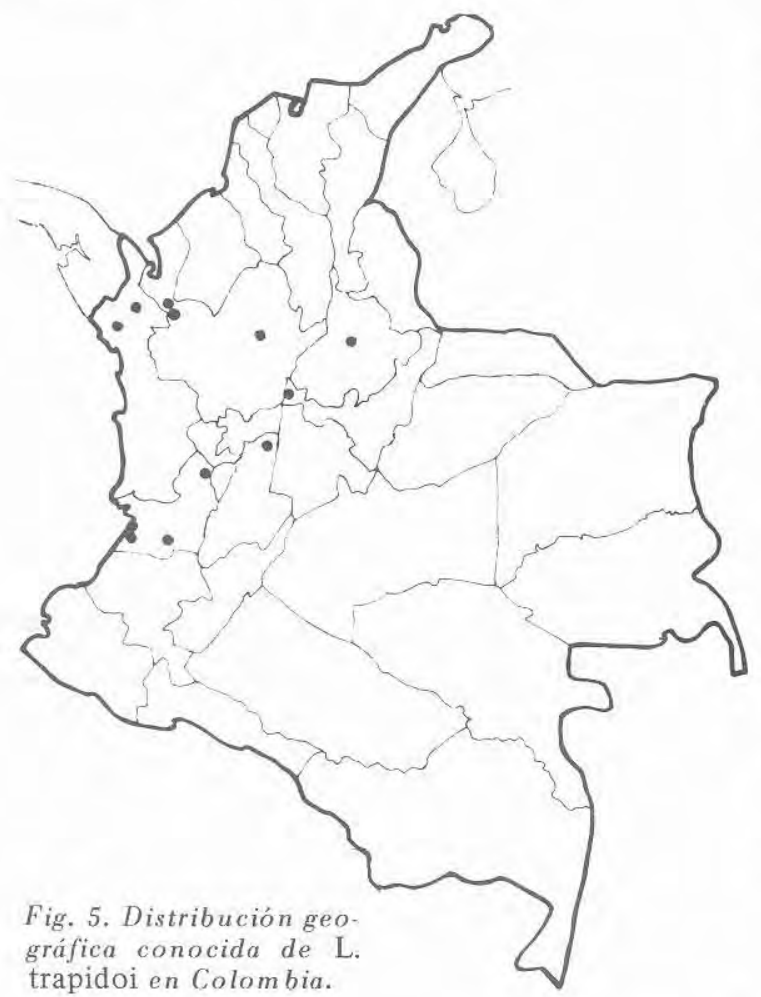

Lutzomyia trapidoi pertenece al subgénero Nyssomyia Barretto, 1962 y dentro de las siete especies de este subgénero encontradas hasta ahora en Colombia, la hembra de .L. trapidoi se distingue fácilmente por la estructura de las espermatecas (Fig. No. 6), pues los anillos de éstas son aproximadamente del mismo tamaño y los ductos comunes son prácticamente inexistentes.

\section{SUMMARY}

In a focus of cutaneous leishmaniasis at the Flor Azul settlement, Mariquita county,
Tolima departament, Colombia, using ligth traps (C D C), Shannon traps, human bait, animal bait (horse) and manual catches of resting sandflies with aspirators were captured a total of $4.081 \delta^{\circ} \delta$ and $7.912 q q$ of Lutzomyia (Diptera, Psychodidae) from February 1979 to May 1981 belonging to nineteen species.

Examination of 3.337 dissected females exhibited promastigotes in 42 out of 2.356 specimens of $L$. trapidoi. The resting 981 females belonged to other different species includling $L$. shannoniand L. gomezi of which one specimen of the former and one from the letter were positive for flagellates.

Thirty two golden hamsters were inoculated intradermally in the nose with promastigotes from thirty L. trapidoi, one $L$. shannoni and one L. gomezi. Three Leishmania strains were isolated from promastigotes of $L$. trapidoi. All the three strains are successfully maintained in hamster and in $4 \mathrm{~N}$ culture medium.

Because of the localization of the flagellates in the midgut of one L. trapidoi infected females and the short incubation period in the hamsters inoculated with the 3 isolated strains and the rapid growth in the culture medium, it is posible to suppose that these strains belong to the Leishmania mexicana complex. It will be necessary to use biochemical methods to characterize and identify these strains of Leishmania.

This is the first time in Colombia, that strains of Leishmania are isolated from sandflies captured using human bait.

\section{AGRADECIMIENTOS}

Los autores desean expresar sus agradecimientos al doctor Luis Arturo Castaño, director del Hospital "San José" de Mariquita, quien siempre nos ha brindado su ayuda. A los señores Eutimio Guerra, Enrique Martínez, Víctor Manuel Ochoa y Rodrígo Lozano por su decisiva ayuda en el trabajo de campo. A la señora Carmenza Herrera de Zambrano, por la transcripción del manuscrito. Al doctor Gabriel Osorno M. por el excelente dibujo que ilustra las estructuras de la hembra de L. trapidoi. 
ALBERTO MORALES, AUGUSTO CORREDOR Y COLABORADORES
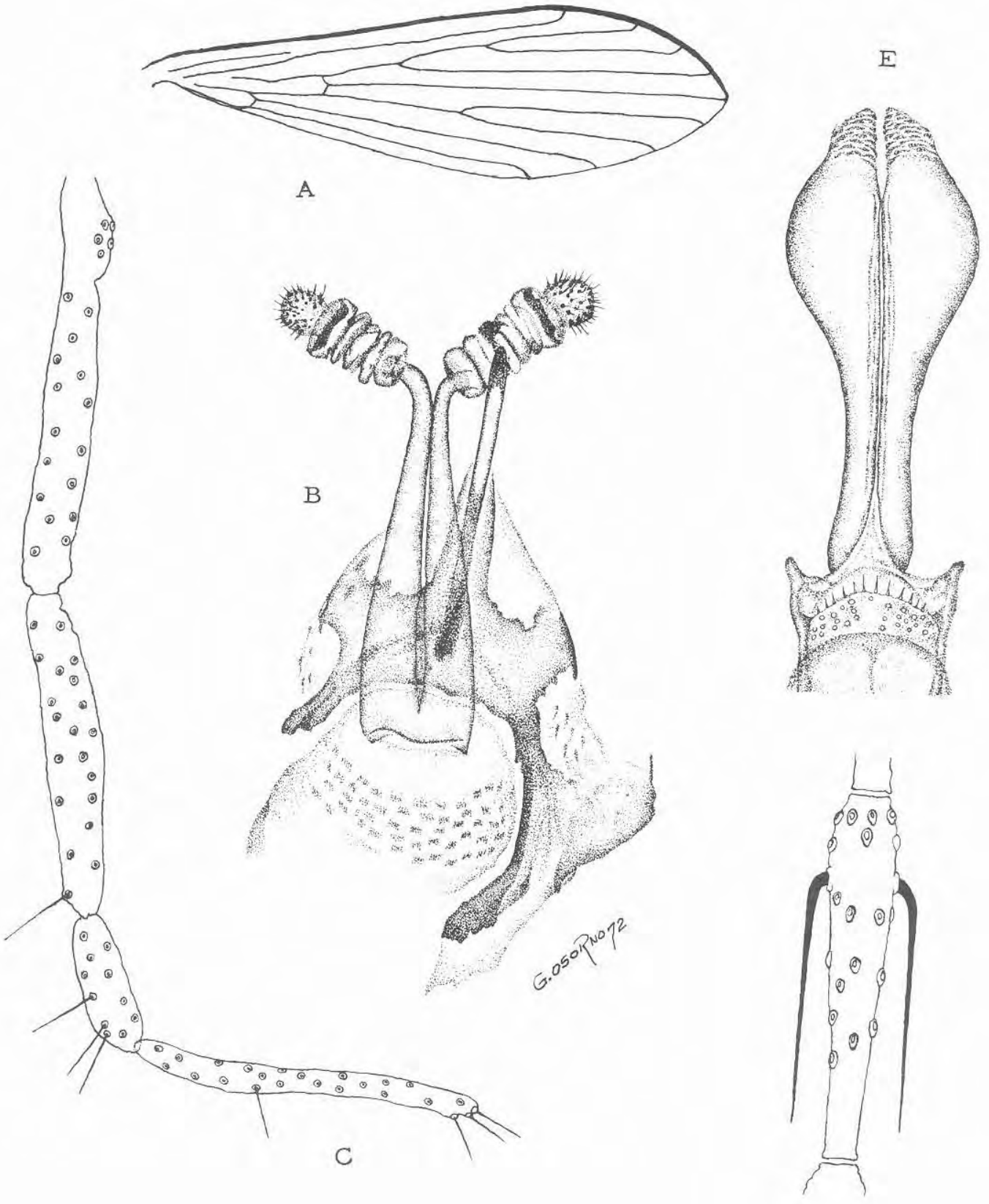

$\mathrm{D}$

Fig. 6. Lutzomyia trapidoi + A.Ala-B. Espermateca C. palpo- D. Ascoide E. Cibario. 


\section{BIBLIOGRAFIA}

1. Espinal S, Zonas de Vida o Formaciones Vegetales de Colombia. Memoria explicativa sobre el mapa ecológico. Instituto Geográfico Agustín Codazzi, 1977, Vol X111 (11).

2. Minter D.M. Three new sandflies (Diptera Psychodidae) from East Africa, with notes on other species. Bull. ent Res, 1963, 54: 483 .

3. Lane J. The preservation and mounting of insects of medical importance, WHO/VBC/ 74. 502: 1 .

4. Minter, D.M. and Goedbloed, E. The preservation in liquid nitrogen of tsetse flies and phlebotomine sandflies natu. rally infected with trypanosomatid flagellates. Trans. $R$. Soc. Trop. Med. Hyg. 1971, 65 (2): 175.

5. Lainson, R., Ward, R.D. and Shaw, J.J. Leishmania in phlebotomid sandflies : VI. Importance of hindgut development in distinguishing between parasites of the Leishmania mexicana and L. braziliensis complexes. Proc. R. Soc. London. B. 1977, 199: 309.
6. MC. Connell, E. Leptomonads of wild-caught Panamanian Phlebotomus: Culture and animal inoculation. Exp. Parasitol. 1963, 14: 123 .

7. Johnson, P. T., MC. Connell, E, and Hertig, M. Natural and experimental infections of leptomonad flagellates in Panamanian Phlebotomus sandflies. J, Parasitol. 1962 48: $15: 8$

8. Barreto P. Artrópodos Hematofagos del Rio Raposo, Valle, Colombia. IV. Psychodidae. Caldasia. 1969, Vol. X (49):459.

9. Osorno-Mesa, E. et al. Phlebotominae de Colombia (Diptera, Psychodidae). IX. Distribución geográfica de especies de Brumptomyia Franca y Parrot, 1921 y Lutzomyia Franca 1924 encontradas en Colombia, S.A. Rev. Acad. Col. Ciencias Exactas, Fisicas y Naturales, 1972, Vol XIV (53) : 45.

10. Young, D.G. A review of the bloodsucking psychodid flies of Colombia (Diptera: Phlebotom inae and Sycoracinae). Agricultural Experiment Station, Institute of Food and Agricultural Sciences, University of Florida, Gainesville F.A. 1979, Bulletin 806, 266 pp. 